\title{
Demineralized bone matrix: A cheap solution for ulna defect healing in a Pigeon
}

Ahmed Tunio $^{1 *}$, Jalila $\mathrm{Abu}^{2}$, Dildar Hussain Kalhoro ${ }^{3}$, Asmatullah $\mathrm{Kaka}^{3}$, Shahid Hussain $\mathrm{Abro}^{3}$, Huma Rizwana ${ }^{3}$ and Shazia Parveen Tunio $^{4}$

1. Department of Surgery and Obstetrics, Faculty of Veterinary Sciences, Sindh Agriculture University TandojamPakistan

2. Faculty of Veterinary Medicine, University Putra Malaysia-Malaysia

3. Faculty of Veterinary Sciences, Sindh Agriculture University Tandojam-Pakistan

4. Pakistan Agriculture Research Council, SSRI, Tandojam-Pakistan

*Corresponding author's email: ahmedtuniodvm2009@gmail.com

Citation

Ahmed Tunio, Jalila Abu, Dildar Hussain Kalhoro, Asmatullah Kaka, Shahid Hussain Abro, Huma Rizwana and Shazia Parveen Tunio. Demineralized bone matrix: A cheap solution for ulna defect healing in a Pigeon. Pure and Applied Biology. Vol. 5, Issue 4, pp1334-1342. http://dx.doi.org/10.19045/bspab.2016.50160

Received: 03/08/2016 Revised: 28/11/2016

Accepted: 04/12/2016

Online First: 10/12/2016

\section{Abstract}

Purpose of this study was to find out whether demineralized bone matrix (DBM) could be used for ulna defect healing in pigeons. For this study total 12 pigeons were purchased then divided into three groups and placing four birds per group. Study duration was 3, 6 and 12 weeks post bone grafting. A 1-cm bone defect was created in left ulna only then DBM graft was placed in between the ulna defect and graft was covered with host tissue. Surgical wound was closed with Vicryl 30 suture material. Then ulna fracture was fixed with four external skeletal fixation pins (type-1). The DBM graft was prepared from donor pigeon bone using hydrochloric acid (0.6M). DBM bone graft healing was evaluated with radiography and histology techniques. Results of radiographic assessment showed no bone graft healed after 3 and 6 weeks post-surgery, but after 12 weeks of healing, 50\% birds showed bone union. While, histological observations showed DBM graft was successfully incorporated in defect site and formed some new bone formation after 3 and 6 weeks of surgery. After 12 weeks of graft healing there was increased new bone formation and good graft incorporation. DBM graft is easy to prepare and could be useful for defect healing in birds because it have bone morphogenic proteins (BMP-2). This study investigated that DBM graft showed potential effect for new bone formation and best graft for defect healing in pigeon.

Keywords: Pigeon; Allograft; Demineralized bone matrix; Bone union; Bone formation

\section{Introduction}

Most of the bone grafts are tested on animal model in medical and veterinary research. Rat, rabbit mouse and birds are commonly used as animal models. Ultimate goal of the bone graft study is to solve the bony defect problems in human and animal patients. Demineralized bone matrix (DBM) is a bone graft material used for reconstruction of bone defect healing. Segmental defect in birds is an orthopaedic problem and bony segment loss will not heal unless if treated by bone 
graft substitute. However, filling of bony defect could help to reduce the gap and provide environment for faster fracture healing. In bone grafting autogenous bone graft is considered as "gold standard" for repair of bone defects [1]. But harvesting of the autogenous bone graft from another donor site is a problem. Therefore, DBM graft plays a key role in the replacement of allograft and is a best choice for defect healing [1]. DBM is one of the bio-materials is used for bone formation [2]. It is prepared in powder, chipped and tubular form that has been used in animal and in bird model experiments [3-4]. It is a cheap bone graft and prepared by removing mineral from bone using hydrochloric acid $(0.6 \mathrm{~N})$ and remaining matrix has ability to induce new bone formation [5]. When DBM graft is implanted in defect site, healing occurs by three biological process such as ostogenesis, osteoinductionand osteoconduction [6]. The osteoinductive property of DBM supports the undifferentiated mesenchymal cells into the osteoblast cells, osteoconduction provide frame work and osteogenesis is the process of bone generation from bone forming cells [7]. It is well known that fracture repairing is a challenge in birds [6] and there is limited literature on the use of bone grafts in birds. The main reason for a bone graft study is to provide the best treatment in cases of bone fragment loss due to fracture in birds. It is useful bone graft substitute because it induces new bone formation and provides mechanical support for fracture healing [8]. With the implantation of DBM in defect site it reduces the healing time. Many studies reported that DBM provides a framework for bone formation and also advances the differentiation of mesenchymal cells into new bone forming cells by a five-day process [9-10]. DBM is used in humans for bone graft surgery [11]. Furthermore, in birds the bones such as the humours and femur are pneumatised because of such a unique structure, the bones are brittle and therefore many birds are at risk of fractures [12]. In short, the sources of autogenous bone grafts in birds are limited [13]. On the source of mentioned results in various animals, it was decided to conduct an experiment on pigeons using DBM. Thus, DBM is one of the suitable bone graft for avian bone defect healing [4, 14] which has potential for bone defect repair. On the basis of the above reported facts it was decided to investigate the new bone formation capability of avian DBM graft for ulna defect healing in pigeons. It was hypothesized that DBM could be most suitable bone graft substitute for bird bone defect healing. Therefore, DBM implanted in a pigeon ulna defect. Bone graft healing was assessed by radiology and histology. Results of this study would be helpful for surgical management of bony defects using DBM graft in domestic and wild birds.

\section{Methodology}

This experimental study was conducted in the Department of Veterinary Clinical Studies, Faculty of Veterinary Medicine, University Putra Malaysia. The processed demineralized bone matrix (DBM) of avian source was tested using adult pigeons of both sex and ages ranging from 5-6 months old and mean body weight 295.5 gram body weight were used in this study. They were kept in an experimental house and two birds housed in a cage. Every day all birds were fed with commercial bird feed and water ad libitum. Food intake and water consumption was not recorded. During the study birds were ringed in legs and numbered from 1 to 12 . All birds received thoroughly physical examination and observed daily for any disease. The experimental protocols performed on all pigeons were reviewed and approved by the animal care and use committee of the Faculty of Veterinary Medicine, University Putra Malaysia under approval number 10R118. 


\section{Preparation bird deminaralized bone matrix (DBM)}

For this study bone graft study DBM was prepared by sacrificing the two adult pigeons using I/V injection of sodium pentobarbital, where long bones were aseptically removed from carcass of pigeons. The collected bones were cleaned with sterilized distilled water and frozen at $-80^{\circ} \mathrm{C}$ for two days. All bones were washed then defatted in ethanol and decalcified in hydrochloric acid (HCL) $0.6 \mathrm{M}$ for three days with daily change. When $\mathrm{pH}$ of the decanting acid was lower than 0.9 then demineralized bones were removed from acid and bones were cut into $1-\mathrm{cm}$ in a tubular shape. In next, DBM grafts were washed in de-ionized water for 5-minutes and neutralized in phosphate buffered saline to maintain the $\mathrm{pH}[6]$. DBM grafts were kept in sterile glass vials and lyophilized under freeze-drying machine and then stored at $6{ }^{\circ} \mathrm{C}$ till next application $[8,14,15]$.

\section{Surgical procedure on birds}

For this study total 12 pigeons were randomly divided into three groups with four birds in each. While, ulna defects were implanted with a DBM graft only and graft healing was evaluated at 3,6 and 12 weeks postoperatively. Anesthesia was performed in all birds using Isoflurane with the combination of oxygen. A face mask was used and oxygen was given first for 2 minutes afterwards general anesthesia was induced with 5\% Isoflurane and oxygen flow of one litre /minute through a non-breathing circuit system. Then 2-mm sized un-cuffed

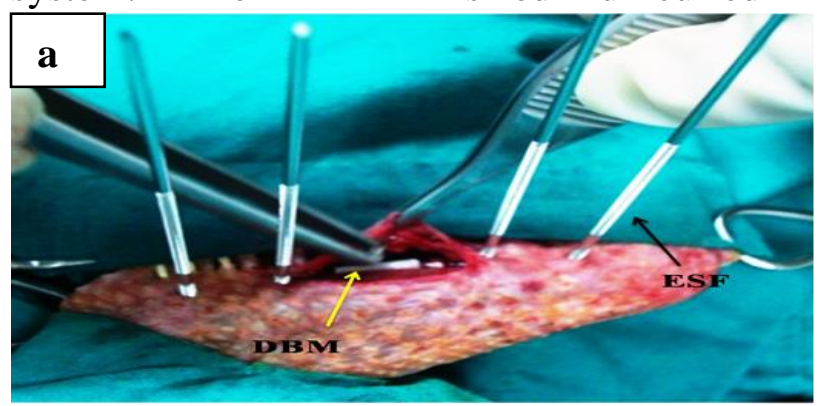

endotracheal tube (Cole-type) was placed in trachea of the birds. After intubation aesthesia was maintained at $2.5 \%$ concentration of Isoflurane and mixed with Oxygen at 0.5 liters/minute [16]. After anesthesia feathers were plucked around left ulna and operative area was scrubbed using chlorexidine solution and 10\% Povidoneiodine.

All pigeons were placed on dorsal recumbency and covered with surgical drape and operative site was exposed. All bones were approached dorsally to expose the ulna bone and similar procedure was performed by [17]. A 2-cm longitudinal skin was incised and ulna bone located. After exposure of ulna bone a $1-\mathrm{cm}$ ulna defect was made at mid shaft in each bird and fracture was fixed with four external skeletal fixations (ESF) positive profile threaded 0.045" size pins. All ESF pins were inserted on one side of ulna using Jecobs chuck. After fracture fixation DBM graft $(1-\mathrm{cm})$ tubular piece was implanted in between the defects (Figure 1a). Lastly, skin and muscles was sutured with Vicry $3 / 0$ in a simple interrupted pattern. Subsequently a Penrose tube $10-\mathrm{cm}$ long was inserted over the ends of the ESF pins and tube was filled with acrylic mixture (acrylic powder 10 gram and5 $\mathrm{ml}$ acrylic liquid; Jorvet ${ }^{\mathrm{TM}}$, Jorgensen, Inc., USA). Afterwards, Trans ESF pins over the acrylic column was then cut one by one using a pin cutter (Figure 1b). The skin wound was covered with figure-of-eight bandage [4].

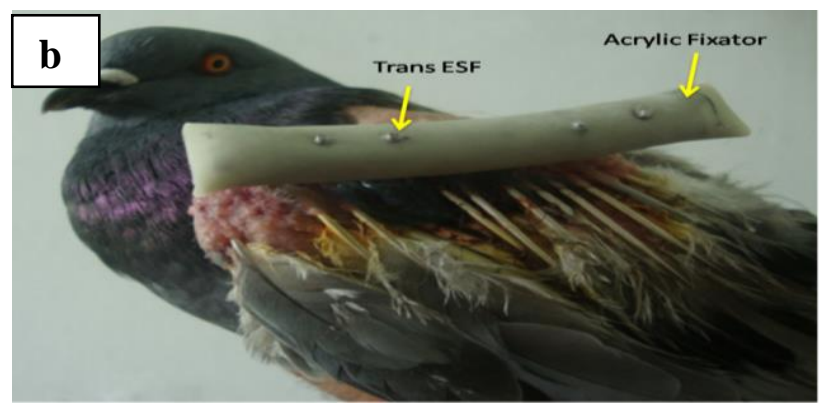

Figure 1. (a) Arrow shows implanted DBM graft in between the ulna defect of a pigeon (b) Postoperative photograph shows acrvlic column and Trans ESF pins were cut in a pigeon ulna 


\section{Post-operative care}

Post-surgery birds were kept in animal house in a separate cage, allowed them to eat and drink ad labium. Figure of eight bandage was checked and changed every day. For first week diluted povidone-iodine was placed on incision site and antibiotics 5\% Baytril (15 $\mathrm{mg} / \mathrm{kg}$ ) given for one week PO. Pin site infection, loosening of ESF pins and bone graft rejections are common complications during healing time [18]. But there was no any complications were found in any birds.
This study evaluated the birds for three endpoints i.e.3, 6 and 12 weeks post-surgery using radiology and histology techniques.

\section{Bone graft healing assessment Radiology}

For all birds radiograph of ulna was exposed on the day of DBM implantation and after every endpoint to assess the defect healing using scoring system. Presence of fracture line was scored as visible or not visible. Radiographic union was considered as a complete bone graft healing (Figure 2).
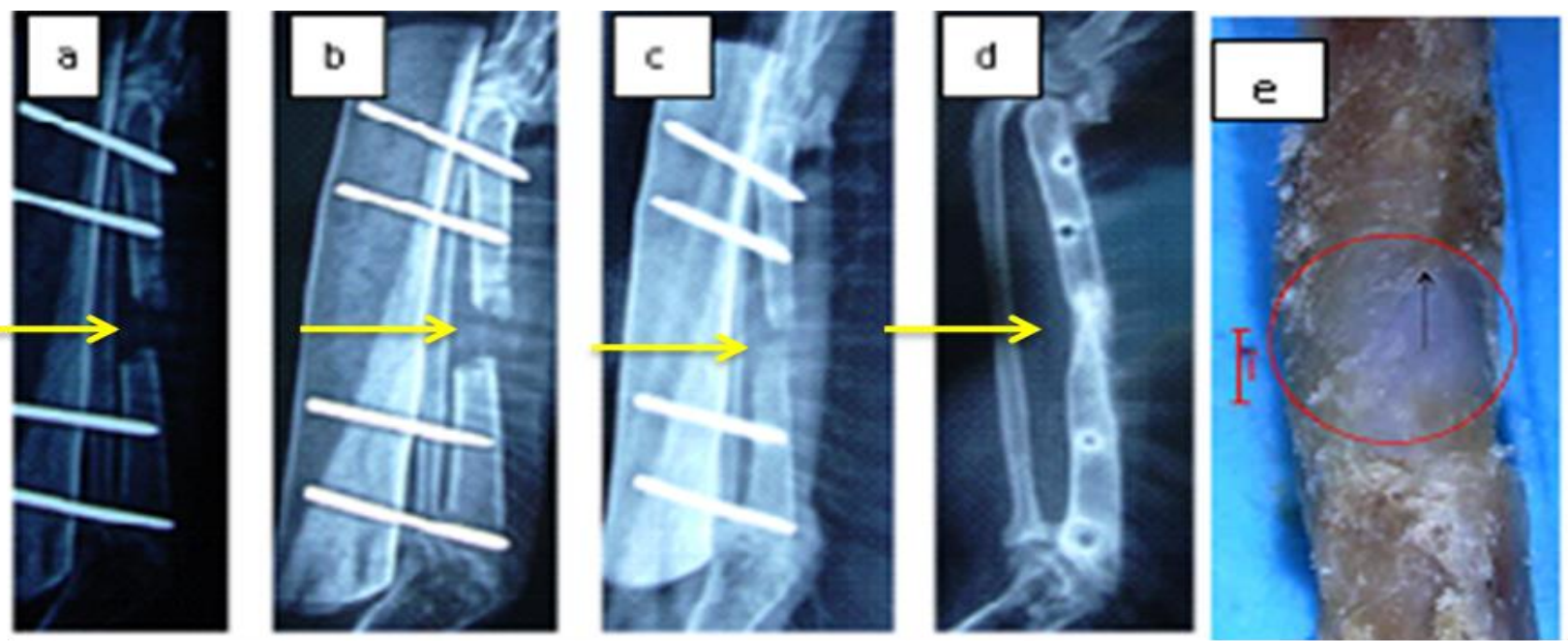

Figure 2. Radiological findings of DBM for pigeon ulna defect healing (a) Postoperative VD view showing there was good bone alignment and reduction with ESF (b) Arrow showing no evidence of bone union at 3-weeks post-surgery (c) Arrow showing some evidence of callus formation and extension of new bone at 6-weeks post-surgery (d) Arrow showing visible evidence of bone union at 12-weeks post-surgery and ESF removed (e) Circle showing complete graft attachment with host and bone union

\section{Post-mortem and histology}

For histological examination birds were euthanized using I/V injection of sodium pentobarbital and post-mortem examination was conducted. Implant was movable at 3 and 6 weeks of healing. However, ulna was stable in 12 weeks period of healing. Bone graft along with soft tissue covering were removed from sacrificed birds and processed for histology. All specimens were fixed in $10 \%$ neutral buffered formalin solution for four days on daily change [19] then decalcified using 5\% formic acid for three days with daily changes till complete removal of calcium [14]. Decalcified bones were processed in an automatic tissue processor and embedded in paraffin. Then multiple 5- $\mu \mathrm{m}$ thickness sections were cut using microtome from each specimen [20]. The sections on slide were stained with haematoxylin and eosin (H\&E) stain. Histological assessment of each specimen was confirmed under light microscope, where presences of the quality of union, cortex development and bone graft incorporation were recorded [3].

\section{Statistics}

The results from radiological and histological data were expressed as means and standard errors ( \pm SE) from all birds and then 
compared by Kruskal-Wallis non-parametric ANOVA. All the tests were conducted at 95\% confidence level and statistical analysis was conducted using SPSS $21.0 \mathrm{v}\left(\right.$ IBM $^{\circledR}$ SPSS ${ }^{\circledR}$ Inc., USA).

\section{Results}

Present study was conducted on 12 birds for the duration of (3, 6 and 12 weeks) postoperative time. There was no any bird expired during observation time and no bird also showed DBM graft rejection.

\section{Radiology findings}

Radiographic results of defect healing (Table 1) showed that none of the birds showed bone union during 3 and 6 weeks of study. There was no significant difference found in radiological parameters in between any bird ( $p>0.05$ ). Radiographic examination showed a radiolucent appearance in between defects and defect remained empty. As shown Figure 2d, DBM graft healing after 12 weeks, radiological examination have showed (2/4) birds showed clinical union and remaining two $(50 \%)$ birds have no sign of clinical union. There was a significant difference of defect healing with DBM at 12 weeks $(\mathrm{P}<0.05)$ as to compare 3 and 6 week respectively.

Table 1. Radiological results of ulna defect healing with DBM in pigeon

\begin{tabular}{|l|l|l|l|}
\hline \multirow{2}{*}{ Parameters } & \multicolumn{3}{|c|}{ Time period of healing (Mean \pm SE) } \\
\cline { 2 - 4 } & 3-week & 6-week & 12-week \\
\hline Bone formation & $0.00 \pm 0.00^{\mathbf{x}}$ & $0.00 \pm 0.00^{\mathbf{x}}$ & $1.50 \pm 0.28^{\mathbf{y}}$ \\
\hline Fracture line & $0.00 \pm 0.00^{\mathbf{x}}$ & $0.00 \pm 0.00^{\mathbf{x}}$ & $0.50 \pm 0.50^{\mathbf{y}}$ \\
\hline Callus formation & $0.00 \pm 0.00^{\mathbf{x}}$ & $0.00 \pm 0.00^{\mathbf{x}}$ & $1.25 \pm 0.25^{\mathbf{y}}$ \\
\hline CSD healing & $0.00 \pm 0.00^{\mathrm{x}}$ & $0.00 \pm 0.00^{\mathrm{x}}$ & $2.25 \pm 0.25^{\mathrm{y}}$ \\
\hline
\end{tabular}

$\mathbf{x , y , z}$. Means within column with different superscripts differed due to time $(\mathrm{P}<0.05$

\section{Histology findings}

Histological findings after 3 weeks of DBM healing (Table 2) showed that defect healed with the fibrous connective tissue. A small amount of new cortex had developed and there was some graft incorporation with the host bone. However, there was no significant difference $(p>0.05)$ in terms of quality of union, cortex development and bone-graft incorporation at 3 weeks in any bird (Figure $3 a)$. Over 6 weeks of DBM graft showed advanced defect healing, defect was covered with connective tissue and graft incorporation with the host bone in all birds. The defects healed with fibro-cartilage union and formation of new bone next to the bone graft. The graft was present and the matrix had achieved vascularisation at the defect site. However, there was no significant difference $(p>0.05)$ in conditions of quality of union, cortex development and bone graft incorporation at 6 weeks of study and complete clinical union had not yet been achieved in this group (Figure 3b). After 12 weeks post-surgery period of DBM graft healing (Figure 3c) all the defects showed good bone graft incorporation with the host bone as compared to 3 and 6 weeks graft healing. A few sections revealed a cartilage area and some bone formation in the matrix. Mesenchymal cells had developed on the surface of the tubular graft. Histological findings of present study showed that the defects were healed by the newly developed bone with a trabecular form and DBM were covered with connective tissues, had formed fibro cartilaginous union and there was extension of new bone, and minute quantities of new bone were observed. No significant radiological and histological changes were observed during 3weeks and 6weeks of DBM healing in pigeons. Meanwhile, significant difference $(\mathrm{P}<0.05)$ in terms of the quality of bone union, cortex development and graft 
incorporation was observed after 12 weeks of the study.

Table 2. Histological results of ulna defect healing with DBM in pigeon

\begin{tabular}{|l|l|l|l|}
\hline \multirow{2}{*}{ Parameters } & \multicolumn{3}{|c|}{ Time period of healing (Mean \pm SE) } \\
\cline { 2 - 4 } & 3-week & 6-week & 12-week \\
\hline Quality of union & $1.25 \pm 0.25^{\mathrm{x}}$ & $2.00 \pm 0.00^{\mathrm{y}}$ & $2.00 \pm 0.00^{\mathrm{y}}$ \\
\hline Cortex Development & $1.00 \pm 0.00^{\mathrm{x}}$ & $2.00 \pm 0.00^{\mathrm{y}}$ & $2.00 \pm 0.00^{\mathrm{y}}$ \\
\hline Bone-graft incorporation & $1.00 \pm 0.00^{\mathrm{x}}$ & $2.00 \pm 0.00^{\mathrm{y}}$ & $2.00 \pm 0.00^{\mathrm{y}}$ \\
\hline
\end{tabular}

$x, y$ Means within column with different superscripts differed due to time $(\mathrm{P}<0.05)$

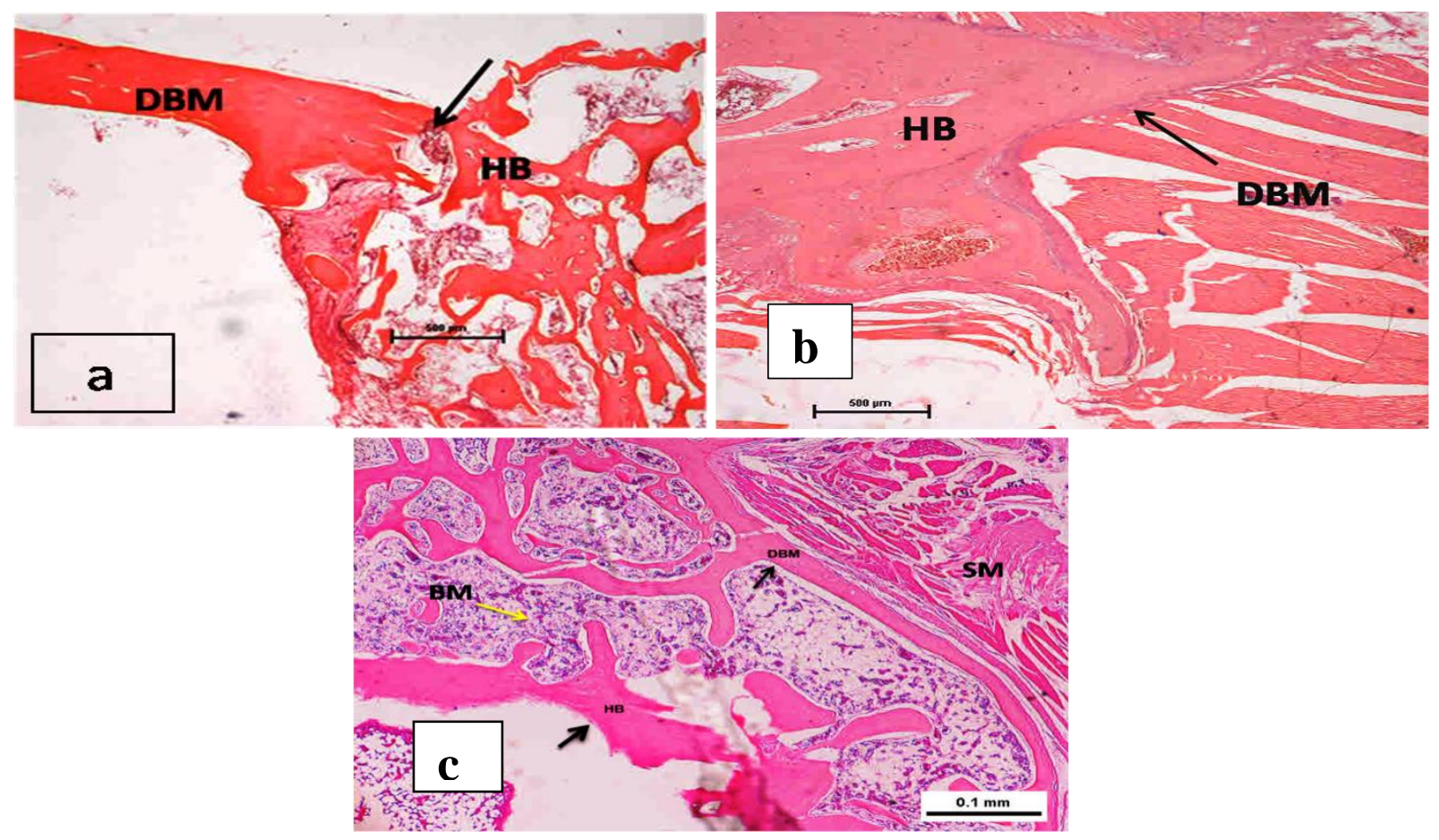

Figure 3. Histological findings of DBM graft healing in a pigeon ulna (a) DBM healing at 3weeks, Arrow showing graft DBM graft incorporation. (b) DBM healing at 6weeks. Arrow showing implant incorporation with host bone (c) DBM graft incorporation at 12 weeks post implantation. Arrow showing DBM graft and new bone formation. $\mathrm{BM}=$ Bone marrow formation in DBM. HB=Host bone, $\mathrm{SM}=$ Skeletal muscle. $(10 \mathrm{x}, \mathrm{H} \& \mathrm{E}$ stain)

\section{Discussion}

Fracture due to trauma is a common surgical problem in domestic and wild birds. In this study demineralized bone matrix (DBM) avian source was used for ulna defect healing. Present study was planned to investigate the DBM and fixed in between the bone defects of pigeon ulna. On the basis of evaluating the DBM for defect healing, this study was divided in three endpoints (3, 6 and 12 weeks) post-operatively. DBM graft has proved to be useful to achieve bone union in birds. This study showed that defect treated with DBM is useful and found a good bone graft substitute on radiological examination after 12 weeks of graft healing. However, there was no significant bone formation found during 3 and 6 weeks post-surgery in any bird. None of the birds showed any inflammatory reaction but improved bone graft healing was observed when the time was extended. There was a significant 
difference in terms of the quality of bone union, cortex development and bone graft incorporation after 12 weeks study duration $(\mathrm{p}<0.05)$. In birds fracture heal faster as compare to mammalian fracture, because of there is increased production of calcium. When fracture happens and bone segment loss occurs due to fracture where defect will not heal until unless not treated with bone graft materials. It is fact that bone graft source is limited in birds, where other bone graft supply is helpful for fracture healing in birds [13]. From which DBM is alternate of autogenous and therefore DBM graft had was filled in 1-cm gap that had enhanced the defect healing. Results from this study revealed that when the duration was prolonged, the bone graft healing was good with bone union and graft incorporation, but no remodelling was observed. Fortunately, avian DBM a cheap graft had showed significant difference for defect healing $(\mathrm{p}<0.05)$ and bone union and bridging were observed at 12 weeks post-grafting. Study by Urist [21] discovered in 1965 and reported that DBM possesses BMPs which induce endochondral ossification. However, the findings of this study are in agreement with a study [14] implanted DBM in a pigeon model and they found bone formation. In the first two weeks after the DBM implantation, the osteoblasts were activated and formed bone through endochondral ossification and bridging [7]. The literature shows that preparation of DBM is very easy and cost effective [15] and thus a similar procedure was used to prepare DBM from avian source in present study. In one study [22] mentioned that fracture is a clinical problem in birds because birds have thin cortex [26] and limited studies has been conducted to assess fracture healing in birds [14]. In this study, the proposed bone graft DBM was prepared and then implanted in the birds, resulting in cortical bone development and good bone graft incorporation. Thus, the results are in agreement with a study [15], where DBM was prepared from rabbits and bones were decalcified with 0.6 Mol HCL. After processing DBM contains BMPs that change cells into new bone formation [23]. Already reported that autogenous bone grafts are considered to be the "gold standard" because they have osteogenesis, osteoinduction and osteoconduction properties [24]. Because of limitations of autografts in birds alternative bone grafts, such as DBM could be used for the repair of bone defects. After 12weeks, almost quality of the bone union, the cortical development, and the graft incorporation were achieved due to the osteoinductive efficacy exerted by the grafted tubular DBM in the pigeon. These findings are supported by [21] they found new bone formation was due to the influence of DBM. In one study [25] disagreed and reported that DBM had no effect on bone formation in skeletal defects in the rabbit study. Meanwhile, we found DBM induced bone formation and cortical development in the pigeon used in present study and is thus useful for the repair of fractures in birds [26]. Beside this [14] found similar histological confirmation for new bone formation at six weeks in pigeon and reported that bone formation occurred via endochondral ossification. Furthermore, DBM encouraged new bone formation and bridging of bone defects in different animal models [8]. Therefore, the results of this study and other results support the fact that DBM has a potential outcome on bone formation and is suitable for the repairing of bone defects in birds after 12 weeks. The benefit of this experiment was that DBM as alone graft prepared from birds had provided cortical bone and good bone graft incorporation in birds. In the present study, the histological results showed that the quality of bone union, cortical development and graft incorporation were achieved due to the osteoinductive efficacy of avian DBM. 


\section{Conclusion}

This study confirmed that DBM graft possess promising effect for novel bone formation and defect healing in pigeon model because it contains bone morphogenic proteins (BMPs). Therefore, potential effect of DBM proved the effectiveness of graft. A further study is required to understand the mechanism of bone graft healing for longer period in animal model.

\section{Authors' contributions}

Conducted the experiment: A Tunio, Designed and financial support for this experiment: J Abu, Edited the manuscript: DH Kalhoro, Assisted in surgery of the birds: A Kaka, Edited and guided for the manuscript: SH Abro, Checked the manuscript: H Reizwana, Helped in the experiment: SP Tunio.

\section{Acknowledgement}

This study has been supported by Dr. Jalila Abu, Associate Professor, Department of Veterinary Clinical Studies, Faculty of Veterinary Medicine, Universiti Putra Malaysia and thank to providing financial support for this study on Pigeons.

\section{References}

1. Laureano Filho JR, Andrade ES, Albergaria-Barbosa JR, Camargo IB \& Garcia RR (2009). Effects of demineralized bone matrix and a'Ricinus communis' polymer on bone regeneration: a histological study in rabbit calvaria. J Oral sci 51(3):451456.

2.Schouten CC, Hartman EH, Spauwen PH \& Jansen JA (2005). DBM induced ectopic bone formation in the rat: the importance of surface area. J Mat Sci. Mat in Med 16(2):149-52.

3.Öztürk A, Yetkin H, Memis L, Cila E, Bolukbasi S \& Gemalmaz C (2006). Demineralized bone matrix and hydroxyapatite/tri-calcium phosphate mixture for bone healing in rats.
International orthopaedics 30(3):147152.

4.Sanaei MR, Abu J, Nazari M, Faiz NM, Bakar MZ \& Allaudin ZN (2011). Heterotopic implantation of autologous bone marrow in rock pigeons: possible applications in avian bone grafting. $J$ Avian Med and Surg 25(4):247-53.

5.Von Versen R, Denner K, Freistedt B, Sehrt B \& Matthes G (1998). A method for the preparation of DBM. Zeitschrift fur medizinische Laboratoriums diagnostic 30 (3): 154158.

6.Bennett RA \& Kuzma AB (1992). Fracture management in birds. J Zoo Wild Med 23:5-38.

7.Jayakumar P \& Di Silvio L (2010). Osteoblasts in bone tissue engineering. Proceedings of the Institution of Mechanical Engineers, Part H: J Eng Medi 224(12):1415-1440.

8.Einhorn TA, Lane JM, Burstein AH, Kopman CR \& Vigorita VJ (1984). The healing of segmental bone defects induced by demineralized bone matrix. A radiographic and biomechanical study. J Bone Joint Surg Am 66(2): 274279.

9.Rabie AB, Deng YM, Samman N \& Hagg U (1996). The effect of demineralized bone matrix on the healing of intramembranous bone grafts in rabbit skull defects. $J$ Dental Research 75(4):1045-1051.

10. Wolfinbarger Jr L, Eisenlohr L \& Ruth K (2008) Demineralized bone matrix: maximizing new bone formation for successful bone implantation. In: Musculoskeletal Tissue Regeneration pp. 93-117. Humana Press.

11. Jin DD (1991). Bone matrix gelatin. Clinical application in 38 cases. Chinese J surgery 29 (5):312-314.

12. Hatt JM (2009). Osteosynthesis in avian surgery with special consideration on 
external fixation. Praktische Tierarzt 90(5): 398-406.

13. Altman RB (1997). Soft tissue surgical procedures. Avian Medicine and Surgery. Philadelphia, PA: WB Saunders 704-32.

14. Jalila A, Redig PT, Wallace LJ, Ogema TR, Bechtold JE \& Kidder L (2004). The effect of chicken, pigeon, and turkey demineralized bone matrix (DBM) implanted in ulnar defects fixed with the intramedullary-external skeletal fixator (IM-ESF) tie-in in pigeons (Columba livia): histological evaluations. The Med J Malaysia 59: 125-26.

15. Tuli SM \& Singh AD (1978). The osteoninductive property of decalcified bone matrix. An experimental study. Bone Joint J 60(1):116-123.

16. Degernes L (2008). Anesthesia for companion birds. Compendium 30(10).

17. Christen C, Fischer I, von Rechenberg B, Flückiger Mand Hatt JM (2005). Evaluation of a maxillofacial miniplate compact 1.0 for stabilization of the ulna in experimentally induced ulnar and radial fractures in pigeons. J Avian Med Surg (3):185-90.

18. Meij BP, Hazewinkel HA \& Westerhof I (1996). Treatment of fractures and angular limb deformities of the tibiotarsus in birds by type II external skeletal fixation. J Avian Med Surg 153-162.

19. Huddleston PM, Steckelberg JM, Hanssen AD, Rouse MS, Bolander ME \& Patel R (2000). Ciprofloxacin inhibition of experimental fracture- healing. $J$ Bone Joint Surg Am 82(2):161-73.

20. Savaridas T, Wallace RJ, Muir AY, Salter DM \& Simpson AH (2012). The development of a novel model of direct fracture healing in the rat. Bone and Joint Research 1(11):289-296.

21. Urist MR, Behnam K, Kerendi F, Raskin $K$, Nuygen TD, Shamie AN \& Malinin TI (1997). Lipids Closely Associated with Bone Morphogenetic Protein and Induced Heterotopic Bone Formation. With Preliminary Observations of Deficiencies in Lipid and Osteoinduction in Lathyrism in Rats. Connective tissue research 36(1): 9-20.

22. Redig PT, Suzuki Y, Abu J \& Jones R (2001). Management of orthopedic problems of the avian forelimb. In Proc Annu Conf Assoc Avian Vet pp 311322.

23. Kalfas IH (2001). Principles of bone healing. Neurosurgical focus 10 (4):14.

24. Pieske O, Wittmann A, Zaspel J, Löffler T, Rubenbauer B, Trentzsch H \& Piltz $S$ (2009). Autologous bone graft versus demineralized bone matrix in internal fixation of ununited long bones. $J$ Trauma management outcomes 15, 3(1): 1.

25. Aspenberg P, Kälebo P \& Albrektsson T (1998). Rapid bone healing delayed by bone matrix implantation. Int J Oral \& Maxillofacial Implants. 3(2):123-127.

26. Swartz SM, Bennett MB \& Carrier DR (1992). Wing bone stresses in free flying bats and the evolution of skeletal design for flight. Nature 359: 726-729. 